\title{
Musculoskeletal Pains and Their Risk Factors in Endoscopists
}

\author{
Gi-young Park \\ Department of Rehabilitation Medicine, Catholic University of Daegu School of Medicine, Daegu, Korea
}

See "Ergonomic Injuries in Endoscopists and their Risk Factors" by Lubna Kamani, Hamid Ali Kalwar, on page 356-362.

Work-related musculoskeletal disorders (WRMDs) are injuries of the muscles, tendons, ligaments, joints, nerves, and spinal discs, including tendinitis, carpal tunnel syndrome, degenerative cervical and lumbar spine disease, and tension neck syndrome. WRMDs manifest as acute or chronic pain that can result in absence from duty, low productivity, increased health care visits, and disability. They are associated with exposure to risk factors in the workplace, such as forceful repetitive tasks, non-neutral long-term postures, abnormal movements, high pressure, and poor instrumentation design. In particular, procedural physicians and surgeons are at a high risk of WRMDs that may be harmful to their lives and influence the quality and quantity of patient care. ${ }^{1}$ Gastroenterologists performing endoscopy are also at a high risk of WRMDs, and the prevalence of endoscopy-related injuries has been reported as $75 \%$ in a previous study. ${ }^{2}$

In this issue of Clinical Endoscopy, Kamani et al. ${ }^{3}$ presented a study on the prevalence of endoscopy-related musculoskeletal pain (ERMP) in endoscopists, comparison of ERMP between endoscopists and non-endoscopists, and the risk factors associated with the pain in endoscopists. They reported that the prevalence of ERMP in endoscopists was higher than that in non-endoscopists ( $95.08 \%$ vs. $54.83 \%)$, which was higher

\footnotetext{
Received: April 20, 2021 Revised: May 1,2021

Accepted: May 1, 2021

Correspondence: Gi-young Park

Department of Rehabilitation Medicine, Catholic University of Daegu School of Medicine, 33, Duryugongwon-Ro 17-gil, Nam-Gu, Daegu 42472, Korea Tel:+82-53-650-4492, Fax: +82-53-622-4687, E-mail: parkgy@cu.ac.kr ORCID: https://orcid.org/0000-0003-0056-1556

(c) This is an Open Access article distributed under the terms of the Creative Commons Attribution Non-Commercial License (http://creativecommons.org/ licenses/by-nc/3.0) which permits unrestricted non-commercial use, distribution, and reproduction in any medium, provided the original work is properly cited.
}

than that reported in other studies. ${ }^{2}$ This discrepancy might be due to the different study designs or different questionnaires used. The authors did not describe whether non-endoscopists were involved in other gastroenterological interventional procedures other than endoscopy. The prevalence of WRMDs in surgeons and interventionalists is high. ${ }^{1}$ Therefore, ERMP in endoscopists should be compared to musculoskeletal pain in non-endoscopists who perform diagnostic or interventional procedures.

The most common injuries in surgeons and interventionalists are cervical spine disorder, followed by rotator cuff disorder, lumbar spine disorder, and carpal tunnel syndrome. The sites commonly affected by ERMP are the thumb, followed by the neck, hand/fingers, lower back, shoulder, and wrist. ${ }^{2}$ Interestingly, the most prevalent sites of ERMP were different between experienced endoscopists (shoulder) and beginners (fingers). ${ }^{4}$ Kamani et al. ${ }^{3}$ also reported that the common sites of ERMP were the lower back (41\%), legs (25\%), hands (23\%), thumb (21\%), and neck (13\%) in endoscopists, and the thumb (26\%), shoulder (21\%), and neck (19\%) in non-endoscopists. These pain sites are related to shoulder, wrist, and hand movements during repeated manipulation of heavy endoscopic equipment for long periods, and incorrect neck and lower back posture while standing and watching the monitor.

Severe ERMP was reported with an increase in the time spent per week performing the procedure. ${ }^{5}$ The time spent performing endoscopy per week and years were independently associated with ERMP. ${ }^{2}$ Other factors that contributed to ERMP were manipulation of the endoscopic parts and prolonged standing. ${ }^{6}$ However, Kamani et al. ${ }^{3}$ reported that factors such as age, frequency of endoscopy, total endoscopic time, and years spent performing the procedure were not significantly associated with ERMP. This result is contrary to that of 
other studies and should be interpreted carefully. The authors explained that the pain might have prevented endoscopists from performing more endoscopic procedures. However, it would be difficult to identify the factors associated with ERMP because most enrolled endoscopists have complained of pain regardless of the number of endoscopies performed and the time spent on the procedure. The other reason for this discrepancy may be the small number of endoscopists enrolled in the study.

Conservative treatments, including medications, massage, physical therapy, rest, splinting, steroid injection, and acupuncture/chiropractic, are commonly used for ERMP. ${ }^{2}$ Kamani et al. ${ }^{3}$ reported that most endoscopists with musculoskeletal pain preferred conservative treatment, except for oral medication, physical therapy, and splinting. In contrast, approximately half of the non-endoscopists chose no treatment. It is necessary to describe the type of conservative treatment that the endoscopists received and explain their preference for this form of treatment. Additionally, further explanation is needed as to why non-endoscopists were not treated. As a physiatrist in pain rehabilitation, I believe that endoscopists would benefit from regular exercise, especially core muscle, upper limb strengthening, and endurance exercises. In addition, grasping the endoscope with a palmar grasp, rather than the fingers, to avoid wrist and hand fatigue would be beneficial to prevent the occurrence and relapse of ERMP.

A multifactorial approach is necessary to reduce the risk of endoscopy-related injuries. These include ergonomic endoscopy instrument design and workplace environment, proper procedural technique, regular education regarding injuries, use of assistive technologies such as wireless device, planned rest breaks between procedures to permit stretching of muscles, scheduled breaks during the procedure, regular core mus- cle strengthening exercises, decreased frequency and duration of endoscopy, and maintenance of proper body posture during the procedure. ${ }^{7,8}$

Conflicts of Interest

The author has no potential conflicts of interest.

Funding

None

ORCID

Gi-young Park:

https://orcid.org/0000-0003-0056-1556

\section{REFERENCES}

1. Epstein S, Sparer EH, Tran BN, et al. Prevalence of work-related musculoskeletal disorders among surgeons and interventionalists: A systematic review and meta-analysis. JAMA Surg 2018;153:e174947.

2. Pawa S, Banerjee P, Kothari S, et al. Are all endoscopy-related musculoskeletal injuries created equal? Results of a national gender-based survey. Am J Gastroenterol 2021;116:530-538.

3. Kamani L, Kalwar H. Ergonomic injuries in endoscopists and their risk factors. Clin Endosc 2021;54:356-362.

4. Byun YH, Lee JH, Park MK, et al. Procedure-related musculoskeletal symptoms in gastrointestinal endoscopists in Korea. World J Gastroenterol 2008;14:4359-4364.

5. Ridtitid W, Coté GA, Leung W, et al. Prevalence and risk factors for musculoskeletal injuries related to endoscopy. Gastrointest Endosc 2015;81:294-302.e4.

6. Hansel SL, Crowell MD, Pardi DS, Bouras EP, DiBaise JK. Prevalence and impact of musculoskeletal injury among endoscopists: a controlled pilot study. J Clin Gastroenterol 2009;43:399-404.

7. Shergill AK, McQuaid KR. Ergonomic endoscopy: An oxymoron or realistic goal? Gastrointest Endosc 2019;90:966-970.

8. Harvin G. Review of musculoskeletal injuries and prevention in the endoscopy practitioner. J Clin Gastroenterol 2014;48:590-594. 